\title{
REVISÃo DE LITERATURA Revisão dos métodos empregados na avaliação da dimensão corporal em pacientes com transtornos alimentares
}

Review of methods employed in the assessment of

body size in patients with eating disorders

Bianca Elisabeth Thurm?', Maria Luiza de Jesus Miranda², Fabio Tapia Salzano³ , Raphael Cangelli Filho 3,4, Táki Athanássios Cordás ${ }^{3}$, Eliane Florêncio Gama’

\section{RESUMO}

Objetivo: O presente artigo analisou os métodos de avaliação da percepção da dimensão corporal abordados na literatura científica. Método: Foram utilizadas a palavra-chave "body image" e a combinação desta com os termos "size perception" e "size estimation", nas bases de dados Medline, Bireme, EBSCO e SCOPUS, para o levantamento científico. O período considerado para essas buscas foi de 1975 a 2010. Resultados: Foram encontrados quatro métodos que avaliam e quantificam a percepção dimensional do corpo, aplicando-se um índice de percepção da dimensão corporal. Os métodos diferem em sua forma de execução, utilização de instrumentos, uso ou não de feedback visual por parte do avaliado, utilização de estímulo tátil ou não para gerar a resposta do avaliado. Conclusão: $\mathrm{O}$ Image Marking Procedure (IMP)

\section{Palavras-chave}

Imagem corporal, distorção da percepção, percepção de tamanho, transtorno alimentar.

\section{Keywords}

Body image, perceptual distortion, size perception, eating disorders. mostrou-se apropriado para a avaliação da dimensão corporal nos distúrbios alimentares, pela possibilidade de avaliar o grau de distorção corporal e dos segmentos corporais específicos sem a interferência visual, cognitiva e qualquer referencial externo comparado aos outros testes que mostraram limitações quanto a esses aspectos. Sugere-se que ele seja utilizado em futuras pesquisas que avaliem o aspecto dimensional da percepção corporal.

\section{ABSTRACT}

Objective: This paper analyzed the methods of assessing the perception of body size discussed in scientific literature. Method: The information search was bases on computerized databases from 1975 to 2010 (Medline, Bireme, EBSCO and Scopus). We used the keyword "body image" and the combination of the terms "size perception" and "size estimation" for the scientific survey. Results: There were four methods found to evaluate and quantify the dimensional perception of the body by applying a body perception index. The methods differ in their form of execution, use tools, whether or not visual feedback from the evaluation, use of tactile stimulation or not to generate the response evaluated. Conclusion: The Image Marking Procedure (IMP) was appropriate for the evaluation of body size on eating disorders by the possibility of evaluating the degree of distortion of the body and specific body segments without visual and cognitive interference and any external referent compared to other tests that showed limitations on these aspects. We suggest that the IMP test could be used in future researches to assess the dimensional aspect of body perception.

1 Universidade São Judas Tadeu (USJT), Departamento de Fisioterapia, Laboratório de Percepção Corporal e Movimento Humano da USJT. 2 USJT, Laboratório de Percepção Corporal e Movimento Humano.

3 Universidade de São Paulo, Faculdade de Medicina, Hospital das Clínicas (HC-FMUSP), Ambulatório de Bulimia e Transtornos Alimentares do Instituto de Psiquiatria (Ambulim-IPq-HC).

4 USJT, Departamento de Psicologia.

Endereço para correspondência: Bianca Elisabeth Thurm Universidade São Judas Tadeu, Departamento de Fisioterapia Rua Taquari, 546, Mooca - 03166-000 - São Paulo, SP, Brasil Tel.: (11) 8182-9426/Fax: (11) 3271-8487

E-mail: biancathurm@uol.com.br 


\section{INTRODUÇÃO}

Os transtornos alimentares caracterizam-se por alterações de comportamento alimentar e com etiopatogenia multifatorial, incluindo fatores biológicos, psicológicos, culturais, familiares e genéticos que interagem entre si de modo complexo'.

Os principais transtornos alimentares são a bulimia nervosa e a anorexia nervosa, e uma característica comum nesses transtornos é a grande importância que a pessoa dá à imagem corporal. Nos dois casos, uma imprecisão na percepção do tamanho real do corpo é evidente, fazendo com que pacientes com anorexia nervosa e bulimia nervosa apresentem uma distorção da percepção de sua dimensão corporal no sentido de se perceberam maiores do que realmente são (hiperesquematia) ${ }^{2-4}$.

A percepção do corpo pode ser conceituada a partir de dois componentes distintos: a imagem corporal e o esquema corporal. A imagem corporal corresponde ao componente atitudinal e se refere aos aspectos cognitivos, atribuições, crenças e expectativas do sujeito em relação ao seu corpo e ao estado emocional proveniente dele. Já o esquema corporal refere-se ao componente perceptual que diz respeito à percepção da dimensão do corpo relacionada especificamente ao tamanho e à forma corporal, somado à representação dinâmica da posição dos segmentos corporais e sua relação com o espaço ao seu redor. Depende das múltiplas informações sensório-motoras e de sua integração para o planejamento e execução das ações s.8. $^{5-8}$ A região cortical responsável por esse mecanismo é a área temporoparietal9-12.

Estudos que avaliam a percepção corporal sugerem que a separação desses componentes é considerada como o princípio básico para a compreensão das distorções da percepção corporal em pacientes com transtornos alimentares ${ }^{13-15}$.

Quando se avalia a imagem corporal, o objetivo é identificar a diferença de como o sujeito se vê e como ele gostaria de se ver ou de ser visto pelo outro. Essa avaliação mostrará o nível de satisfação corporal do sujeito ${ }^{16}$ utilizando testes de escala de silhueta, técnicas de distorção de imagem e desenhos. Nesses testes, avalia-se o corpo inteiro ${ }^{6,7,14}$. Outras formas de avaliar a imagem corporal são por meio de questionários e escalas ${ }^{16-18}$.

O objetivo de avaliar a percepção da dimensão corporal é analisar a diferença entre a medida percebida do próprio corpo e a medida real do sujeito; neste caso, avaliam-se as partes do corpo. A diferença entre as medidas torna-se um indicador do grau de distorção da percepção da dimensão corporal ${ }^{16}$.

A avaliação da imagem corporal é largamente utilizada nos estudos clínicos dos transtornos alimentares; já a avaliação clínica do esquema corporal, ou seja, o nível da distorção da percepção corporal, é muito pouco citada ou mesmo utilizada, apesar de os estudos darem ênfase à separação da avaliação dos componentes atitudinal da dimensional. Percebe-se que a sugestão de avaliar o esquema corporal é mais evidente no âmbito teórico. Segundo Hundleby e Borgoin ${ }^{8} \mathrm{e}$ Gardner ${ }^{14}$, existem problemas metodológicos e falta de consenso entre as pesquisas em relação aos métodos usados para avaliação do esquema corporal.

Portanto, o objetivo foi analisar os métodos de avaliação da percepção da dimensão corporal abordados na literatura científica.

\section{MÉTODO}

A busca de artigos científicos para este trabalho de revisão foi realizada a partir da base de dados Medline, Bireme, EBSCO e SCOPUS.

A busca foi conduzida por meio de descritor do DeCs/ MeSH. O descritor utilizado foi "body image", refinado com os termos "size perception" e "size estimation".

Foram incluídos na análise os estudos que utilizaram testes para avaliar o esquema corporal e que faziam uso da aplicação de um índice de percepção corporal (IPC). Desses, foram analisados apenas os estudos experimentais realizados em população adulta, nas línguas inglesa, portuguesa e alemã e que apresentassem transtorno alimentar. O período considerado para essas buscas foi de 1975 a 2010. Foram excluídos os artigos de revisão e metanálise e aqueles relacionados com avaliações do aspecto atitudinal em relação ao corpo, dentre eles os que incluem as técnicas de distorção da imagem por vídeo e computador, distorção do espelho e fotografia, teste do desenho da figura humana, método de avaliação por meio de silhuetas e questionários.

\section{RESULTADOS}

Verificou-se que o descritor "esquema corporal" não constava na listagem do DeCs/MeSH e, dessa forma, optou-se por um descritor mais próximo a este conceito que foi "imagem corporal" ("body image").

A partir do descritor "imagem corporal", foi encontrado um total de 9.022 artigos até a data determinada pelo levantamento bibliográfico (10/10/2010). Para filtrar essa busca, utilizou-se a combinação dos termos "body image" + "size perception", totalizando 399 artigos, e "body image" + "size estimation", totalizando 126 artigos. Desse total, foram selecionados inicialmente 141 artigos referentes à primeira combinação de unitermos e 65 artigos relacionados à segunda. Após a aplicação dos critérios de inclusão e exclusão delimitados por este estudo, chegou-se a 7 e 9 artigos, respectivamente (Tabela 1). Desses, 8 tratavam de anorexia, 1 de bulimia, 2 de anorexia e bulimia e 5 sem patologia e no sexo feminino (Tabela 2). 
Em relação aos descritores encontrados, pôde-se observar que o termo "imagem corporal" é utilizado em todos os artigos revisados, e em segundo lugar aparece o termo "distorção da percepção". Os outros termos encontrados e sua ocorrência estão descritos na tabela 3.

Tabela 1. Resultado do levantamento bibliográfico utilizando descritores e unitermos

\begin{tabular}{lccc}
\hline Descritor & $\begin{array}{c}\text { No artigos } \\
\text { encontrados }\end{array}$ & $\begin{array}{c}\text { No artigos que } \\
\text { abordam dimensão } \\
\text { corporal }\end{array}$ & $\begin{array}{c}\text { No artigos } \\
\text { selecionados a } \\
\text { partir dos critérios } \\
\text { estabelecidos }\end{array}$ \\
\hline $\begin{array}{l}\text { Esquema corporal } \\
\text { Imagem corporal }\end{array}$ & 0 & & \\
$\begin{array}{l}\text { Imagem corporal + } \\
\text { percepção de tamanho } \\
\text { Body image + size } \\
\text { perception }\end{array}$ & 14 & 0 & 0 \\
$\begin{array}{l}\text { Body image }+ \text { size } \\
\text { estimation }\end{array}$ & 399 & 141 & 7 \\
\hline
\end{tabular}

Com relação aos métodos empregados, foram encontrados 16 artigos, dentre eles 12 aplicaram o índice de percepção corporal (IPC), que é utilizado para classificar a percepção corporal usando a fórmula tamanho percebido/tamanho real $x$ 100. Nesse caso, os sujeitos que se percebem acima de 100\% são classificados como hiperesquemáticos; os que se percebem abaixo de 100\% são considerados hipoesquemáticos; e os que se percebem 100\%, adequados. Foram encontrados quatro procedimentos que avaliam o esquema corporal, sendo eles: 1. Movable caliper procedure (5 artigos), que utiliza uma barra horizontal com dois pontos luminosos montados num carreto. É solicitado ao sujeito que aproxime ou afaste os pontos luminosos para identificar a largura da parte corporal solicitada (cabeça, ombros, cintura, quadril e perna). A barra é mantida na altura dos olhos; 2. Visual size estimation procedure (1 artigo), que se utiliza de uma barra com duas lâmpadas acopladas e, a partir da projeção da luz na parede, o sujeito orienta o avaliador a parar de mover os pontos luminosos quando estes corresponderem à largura do segmento corporal solicitado (largura entre os ossos zigomáticos, entre os dois ombros, abdome, cintura e quadril).

Tabela 2. Características dos artigos consultados quanto à utilização de testes para a avaliação da dimensão corporal

\begin{tabular}{|c|c|c|c|c|c|c|}
\hline Autor & Ano & Pais & $\mathrm{N}$ & Teste utilizado & IPC & Tipo análise \\
\hline Askevold & 1975 & Noruega & $\begin{array}{c}15 \mathrm{AN} \\
22 \text { diversos }\end{array}$ & IMP & Não & Quanti \\
\hline pierloot et al. & 1976 & Inglaterra & $\begin{array}{l}31 \mathrm{AN} \\
20 \mathrm{NL}\end{array}$ & $\begin{array}{l}\text { IMP } \\
\text { MCP }\end{array}$ & Sim & Quanti \\
\hline whitehouse et al. & 1986 & Inglaterra & $22 \mathrm{BN}$ & IMP & Sim & Quanti \\
\hline fichter et al. & 1986 & Inglaterra & $21 \mathrm{AN}$ & $\begin{array}{l}\text { MCP } \\
\text { IMP }\end{array}$ & Não & Quanti \\
\hline whitehouse et al. & 1988 & Inglaterra & $\begin{array}{l}12 \mathrm{AN} \\
20 \mathrm{NL}\end{array}$ & IMP & Sim & Quanti \\
\hline thompson et al. & 1989 & Estados Unidos & 32 NL fem & MCP & Sim & Quanti \\
\hline Bowden et al. & 1989 & Inglaterra & $\begin{array}{l}12 \mathrm{AN} \\
12 \mathrm{BN} \\
24 \mathrm{NL}\end{array}$ & $\begin{array}{l}\text { IMP } \\
\text { VSEP }\end{array}$ & Não & Quanti \\
\hline fonagy et al. & 1990 & Inglaterra & $\begin{array}{l}20 \mathrm{NL} \text { masc } \\
20 \mathrm{NL} \text { fem }\end{array}$ & MCP & Sim & Quanti \\
\hline Thomas et al. & 1991 & Estados Unidos & $200 \mathrm{NL}$ fem & IMP & Não & Quanti \\
\hline lautenbacher et al. & 1992 & Inglaterra & $\begin{array}{l}21 \mathrm{AN} \\
20 \mathrm{NL}\end{array}$ & $\begin{array}{l}\text { IMP } \\
\text { KSEA }\end{array}$ & Sim & Quanti \\
\hline lautenbacher et al. & 1993 & Inglaterra & $42 \mathrm{NL}$ & $\begin{array}{l}\text { IMP } \\
\text { KSEA }\end{array}$ & Sim & Quanti \\
\hline Hundleby et al. & 1993 & Canadá & $100 \mathrm{NL}$ fem & MCP & Não & Quanti \\
\hline molinari & 1995 & Italia & $\begin{array}{l}20 \mathrm{AN} \\
20 \mathrm{NL}\end{array}$ & IMP & Sim & Quanti \\
\hline deter et al. & 1997 & Alemanha & $\begin{array}{l}50 \mathrm{AN} \text { fem } \\
05 \mathrm{AN} \text { masc }\end{array}$ & IMP & Sim & Quanti \\
\hline Lautenbacher et al. & 1997 & Inglaterra & $57 \mathrm{AN}$ & $\begin{array}{l}\text { IMP } \\
\text { KSEA }\end{array}$ & Sim & Quanti \\
\hline Aranda et al. & 1999 & Espanha & $\begin{array}{l}25 \mathrm{BN} \\
19 \mathrm{AN}\end{array}$ & IMP & Sim & Quanti \\
\hline
\end{tabular}

AN: anorexia nervosa; BN: bulimia nervosa; fem: sexo feminino; masc: sexo masculino; NL: normal; esquizo: esquizofrenia; OB: obeso; IMP: image marking procedure; KSEA: kinesthetic size estimation apparatus; MCP: movable caliper procedure; VSEP: visual size estimation procedure; quanti: análise quantitaviva; IPC: quanto ao uso ou não do cálculo. 
Tabela 3. Descritores utilizados pela base de dados dos artigos selecionados

\begin{tabular}{lc}
\hline Descritor utilizado & Ocorrência \\
\hline Imagem corporal & 16 \\
Distorção da percepção & 9 \\
Constituição corporal & 4 \\
Resultado de tamanho & 3 \\
\hline
\end{tabular}

A barra é mantida na altura dos olhos durante o teste; 3 . Kinesthetic size estimation apparatus - KSEA (3 artigos), uma barra com dois puxadores é colocada à altura dos ombros do sujeito com os olhos vendados e ele deve posicioná-los na largura dos pontos corporais correspondentes tocados pelo avaliador (ombros, cintura, quadril e coxa). 4. Image marking procedure - IMP (13 artigos), na qual os sujeitos, com os olhos vendados, projetam numa folha de papel pendurada na parede à sua frente a percepção da dimensão de segmentos corporais determinados, estimulados de forma tátil (alto da cabeça, ombros, cintura, quadril, coxa) (Tabela 2).

\section{DISCUSSÃO}

A busca da literatura científica na base de dados mostrou que não existe uma padronização dos descritores e palavras-chave em relação ao conteúdo dos artigos no que diz respeito à percepção da dimensão corporal e/ou avaliação do esquema corporal. Essa variedade de descritores dificulta a busca específica dos artigos.

O termo imagem corporal sempre foi usado englobando tanto o aspecto atitudinal quanto o dimensional da percepção corporal. Somente por volta de 1990 alguns estudos como os de Thompson e Dolce ${ }^{19}$ e outros ${ }^{14,20}$ começaram a abordar a questão de utilizar avaliações diferentes para cada aspecto da percepção do corpo. Esses estudos foram classificados em dois tipos: as avaliações de corpo inteiro, que estão relacionadas com o componente atitudinal, e as avaliações de partes do corpo, que analisam o componente perceptual da dimensão corporal. As pesquisas sobre avaliação do esquema corporal foram conduzidas principalmente no período entre 1975 e 1989.

Desse período até 2010, a avaliação da dimensão corporal foi utilizada cada vez menos e observou-se que houve uma tendência de as pesquisas relacionadas aos transtornos alimentares focarem mais no aspecto psicológico da doença, mesmo nos estudos utilizando a ressonância magnética para avaliar a ativação e lesões das áreas corticais nesse grupo de sujeitos ${ }^{14-16,18,21}$. Pelo fato de os sujeitos com transtornos alimentares serem assistidos principalmente por uma equipe de psiquiatras e psicólogos, parece natural que os pesquisadores foquem no aspecto atitudinal da percepção da dimensão corporal utilizando instrumentos que estão relacionados à sua área de atuação profissional, como é o caso dos questionários e avaliação da satisfação corporal.

Em relação à falta de padronização dos testes que avaliam a percepção dimensional do corpo, é necessário discutir algumas questões.

Considerando os segmentos corporais utilizados nos artigos para avaliar a dimensão corporal, foi observado que não existe uma padronização. Alguns segmentos como cabeça, ombros, cintura e quadril são avaliados em todos os estudos, porém alguns deles utilizam também a avaliação da percepção das larguras das coxas, pernas e abdome $2,9,19,20$. Em nenhum dos artigos foi mencionado que esses testes que avaliam a percepção da dimensão corporal são validados, dessa forma acredita-se que a falta de padronização da escolha dos segmentos corporais se deva a esse fato, fazendo com que cada pesquisador opte pelos segmentos de acordo com o delineamento de sua pesquisa.

O mesmo ocorre para calcular o tamanho real dos segmentos corporais, ou seja, não há uma uniformização para essa medida. Alguns usam um antropômetro, que é uma régua com hastes deslizantes que avalia grandes segmentos corporais s, $3,13,22-24$, um empregou um compasso de ponta ${ }^{25}$, outros utilizaram um caliper (uma barra) ${ }^{19}$, diversos marcam em uma folha de papel pendurado na parede as medidas reais do sujeito ${ }^{2,92,20,26,27,29,30}$. Os instrumentos utilizados nesses estudos se propõem a medir o tamanho real de forma diferente, podendo gerar diferentes medidas e, desse modo, dificultando a comparação entre os diferentes estudos.

Analisando os testes encontrados, foi observado que todos visam mensurar a capacidade do sujeito em perceber a sua dimensão corporal, e pareceu viável separá-los em: 1. Os que usam uma barra como ferramenta para a avaliação da dimensão corporal; 2. Os que usam o membro superior livre.

Diferenças na forma de execução entre os testes serão apontadas: o uso de uma barra pode ser um diferencial na forma de realização dos testes, pois a barra pode ser um referencial externo que talvez sirva como um guia. Outro ponto importante é que nos testes movable caliper procedure e no visual size estimation procedure, apesar de não informarem claramente nos artigos quanto ao feedback visual, infere-se que os sujeitos permaneceram de olhos abertos. Nesse caso, a visão forneceria informações, possibilitando ao indivíduo fazer ajustes no sentido de comparar a largura real dos segmentos corporais avaliados e a estimativa da percepção da dimensão corporal. Já no IMP, o sujeito marca no papel à sua frente a percepção dos pontos corporais tocados por um avaliador com o membro superior livre. Dessa forma, o teste usa apenas a capacidade do indivíduo de perceber o segmento corporal tocado e, a partir daí, projetá-lo no espaço sem o auxílio da visão. Além disso, não é utilizado nenhum referencial externo, como uma barra, usando somente estí- 
mulos exteroceptivos e proprioceptivos. Desse modo, parece mais adequada a utilização de um teste que não propicie ajustes a partir de referenciais externos (visão ou barra), visto que nos transtornos alimentares a comparação visual com relação ao próprio corpo ou o corpo de outros é distorcida pela própria natureza da doença.

O teste do IMP vem de encontro aos conceitos do esquema corporal, um sistema neurológico que depende de informações exteroceptivas e proprioceptivas para fornecer a capacidade ao sujeito de perceber e reconhecer o corpo sem a utilização da visão ${ }^{9,10,31}$. A utilização de recursos como uma barra e a visão, que ocorre nos demais testes encontrados, acaba favorecendo o ajuste voluntário e automático da percepção da dimensão corporal pelo sujeito.

Nesse sentido, alguns autores sugerem que o IMP é o único método que acessa a percepção da dimensão corporal baseado no estímulo tátil direto, pois o toque sobre a pele promove a base para a percepção dos limites do corpo ${ }^{32}$. As regiões tocadas no IMP correspondem às estruturas anatômicas localizadas nas extremidades corporais como o alto da cabeça, os acrômios na região dos ombros, a cintura e os trocanteres na região do quadril. Somados, estes pontos representam os limites da dimensão corporal. Uma vez que nos transtornos alimentares ocorre uma distorção da percepção dos limites corporais, o IMP consegue identificar o grau da distorção da dimensão corporal a partir do cálculo do IPC. É possível analisar, ainda, qual é a região corporal mais afetada pelo transtorno, já que o teste também avalia partes do corpo que comumente são as maiores queixas dessa população, como as regiões da cintura e do quadril. De acordo com as pesquisas, uma característica importante do teste IMP é que ele permite uma comparação direta da largura percebida e da real e permite calcular valores estatísticos ${ }^{33,34}$.

Apesar de todos os testes encontrados se proporem a avaliar a percepção da dimensão corporal, não se sabe se os instrumentos utilizados levam a um resultado final igual. Um estudo que avaliou a percepção da dimensão corporal em atletas de alto rendimento utilizando dois instrumentos - o KSEA e o IMP - mostrou, a partir da análise estatística de equivalência, correlação negativa entre eles, ou seja, os resultados obtidos são diferentes ${ }^{35}$. Fichter et al. ${ }^{23}$ usaram três métodos para avaliar o distúrbio da percepção corporal em um grupo de anoréxicas, bulímicas e controle: o IMP, o movable caliper procedure e a distorção da imagem por vídeo. Seus resultados mostraram que o IMP foi mais eficiente para discriminar entre pacientes com anorexia nervosa e grupo controle. Os autores consideram que a vantagem do uso dessa avaliação está na simplicidade técnica e conceitual do método.

Segundo Valtolina ${ }^{5}$, o IMP é um método não verbal que envolve a percepção e aspectos da motricidade. A autora utilizou o IMP para avaliar a dimensão corporal e, a partir dos seus resultados, considerou o teste como sendo o mais apropriado, por ser uma forma direta e não verbal de acessar a percepção da dimensão corporal, o que não ocorre quando se usam questionários. O teste de percepção de Askevold foi desenhado para avaliar a percepção das dimensões de diferentes áreas do corpo, fazendo uso da habilidade do paciente de se projetar no espaço. O estudo direto é o mais compatível para esse tipo de investigação, pois tem a vantagem de não precisar se basear em uma percepção não corporal, como é o caso do uso de questionário ou de entrevista ${ }^{23,35}$. O julgamento de valores está muito comprometido nos transtornos alimentares, desse modo o uso de uma técnica direta de avaliação da dimensão corporal leva a crer que minimizaria essa interferência cognitiva.

\section{CONCLUSÃO}

Foram encontrados quatro métodos que avaliam e quantificam a percepção corporal utilizando o índice de percepção da dimensão corporal. Os métodos diferem em sua forma de execução, utilização de instrumentos e o uso ou não de feedback visual por parte do avaliado, uso ou não de estímulo tátil para gerar a resposta do avaliado. Dentre os métodos analisados, o Image Marking Procedure (IMP) mostrou-se apropriado para avaliação da dimensão corporal, particularmente nos transtornos alimentares, por ele ser capaz de ativar a capacidade do sujeito de identificar as partes constituintes do seu corpo e de projetá-lo no espaço sem o auxílio da visão, o que corresponde às funções neurológicas do esquema corporal.

Acreditamos que o teste do IMP possa contribuir para a avaliação do componente perceptual (esquema corporal) e se somar à avaliação do componente atitudinal da percepção corporal tanto na bulimia nervosa quanto na anorexia nervosa, para ampliar a compreensão desse mecanismo complexo que leva à distorção da dimensão do corpo e à busca de abordagem terapêutica direcionada para cada componente.

Levando em conta o aspecto proprioceptivo, a possibilidade de avaliar o grau de distorção corporal e dos segmentos corporais específicos, parece que o IMP é o teste mais adequado para a avaliação do esquema corporal nos transtornos alimentares, por abarcar os aspectos sensoriais da percepção corporal, além da possibilidade de projetar livremente a percepção dos limites corporais sem a interferência visual, cognitiva e qualquer referencial externo comparado aos outros testes que mostraram limitações quanto a esses aspectos.

Sendo a distorção da percepção corporal o sintoma central nos pacientes com transtornos alimentares, não só as estratégias terapêuticas devem ser aprofundadas e desenvolvidas, mas também os métodos de avaliação, de modo a aquilatar os benefícios das terapias empregadas. 
Levando em conta a praticidade, a exequibilidade e o baixo custo do teste de percepção da dimensão corporal (IMP), sugerimos que ele seja utilizado em futuras pesquisas que avaliem o aspecto dimensional da percepção corporal, seja em pacientes com transtornos alimentares ou com síndromes que levam ao distúrbio do esquema corporal.

\section{REFERÊNCIAS}

1. Salzano FT, Aratangy EW, Azevedo AP, Pisciolaro F, Maciel AMB, Cordas TA. Transtornos alimentares. In: Miguel EC, Gentil V, Gattaz WF. Clínica psiquiátrica. Barueri (SP): Manole; 2011. p. 931-52.

2. Lautenbacher S, Kraehe N, Krieg JC. Perception of body size and body satisfaction in recovered anorexic women: comparison with restrained and unrestrained eaters. Percept Mot Skills. 1997;84:1331-42.

3. Fonagy P, Benster R. Adaptive probit estimation and body size: the evaluation of a new psychophysical technique. Brit J Psych. 1990;81:159-71.

4. Aranda FF, Dahme B, Meermann, R. Body image in eating disorders and analysis of its prevalence: a preliminary study. J Psychosomatic Res. 1999;47(5):419-28.

5. Valtolina GG. Body size estimation by obese subjects. Percept Mot Skills. 1998;86:1363-13.

6. Skrzypek S, Wehmeier PM, Remschmidt H. Body image assessment using body size estimation in recent studies on anorexia nervosa. A brief review. Eur Child Adolesc Psychiatry. 2001;10:215-21.

7. Sepúlveda AR, Botella J, Leon JA. Body-image disturbance in eating disorders: a meta-analysis. Psychol Spain. 2002;6(1):83-95.

8. Schwoebel J, Coslett HB. Evidence for multiple, distinct representations of the human body. J Cogn Neurosci. 2005;17:543-53.

9. Lautenbacher S, Roscher F, Strian KM, Pirke J, Krieg JC. Theoretical and empirical considerations on the relation between body image, body schema and somatosensation. J Psychossom Res. 1993;37(5):447-54.

10. Paillard J. Body schema and body image: a double dissociation in deafferented patients. Motor Control. 1999;197-214.

11. Stewart A, Benson P, Michanikou E, Tsiota D, Narli M. Body image perception, satisfaction and somatotype in male and female athletes and non-athletes: results using a novel morphing technique. J Sports Sci. 2003;21:815-23.

12. Medina J, Coslett HB. From maps to form to space: Touch and the body schema. Neuropsych. 2010;48(3):645-54.

13. Hundleby JD, Bourgoin NC. Generality in the errors of estimation of body image. Int J Eat Disord. 1993;1(13):85-92.

14. Gardner RM. Methodological issues in assessment of the perceptual component of body image disturbance. Brit J Psych. 1996;87:327-37.

15. Mussap AJ, Mccabe MP, Ricciardelli LA. Implications of accuracy, sensitivity and variability of body size estimations to disordered eating. Body Image. 2008;5:80-90.
16. Ferrer-García M, Gutiérrez-Maldonado J. Body Image Assessment Software: psychometric data. Behav Res Methods. 2008;40(2):394-407.

17. Cordás TA, Neves JEP. Escalas de avaliação de transtornos alimentares. Rev Psiq Clín. 1999;26(1):ISSN 0101-6083.

18. Mohr HM, Zimmermann J, Roder C, Lenz C, Overbeck G, Grabhorn R. Separating two components of body image in anorexia nervosa using FMRi. Psychol Med. 2009;40(9):1519-29.

19. Thompson K, Dolce JJ. The discrepancy between emotional vs. rational estimates of body size, actual size, and ideal body ratings: theoretical and clinical implications. J Clin Psychol. 1989;45(3):473-8.

20. Lautenbacher S, Thomas A, Roshcer F, Strian KM, Pirke J, Krieg JC. Body size perception and body satisfaction in restrained and unrestrained eaters. Behav Res Ther. 1992;20(3): 243-50.

21. Uher R, Treasure J. Brain lesions and eating disorders. J Neurol Neurosurg Psychiatry. 2005;76:852-7.

22. Pierloot RA, Houben ME. Estimation of body dimensions in anorexia nervosa. Psychol Med. 1978;8:317-24.

23. Fichter MM, Meister I, Koch HJ. The measurement of body image disturbances in anorexia nervosa, experimental comparison of different methods. Br J Psychiatry. 1986;148:443-61.

24. Molinari E. Body size estimation in anorexia nervosa. Percept Mot Skills. 1995;81:23-31.

25. Deter HC, Rolf, H, Herzog W, Muller S. Korperbildstorungen von anorexia-patienten 12 Jahre nach der klinischen Vorstellung. Psychother Phsycosom Med Psychol. 1997;47:1-11.

26. Askevold F. Measuring body image - preliminary report on a new method. Psychother Psychosom. 1975;26:71-7.

27. Bowden PK, Touyz SW, Rodriguez PJ, Hensley R, Beumont PJ. Distorting patient or distorting instrument? Body shape disturbance in patients with anorexia nervosa and bulimia. Br J Psychiatry. 1989;155:196-201.

28. Whitehouse AM, Freeman CL, Annandale A. Body size estimation in bulimia. Br J Psychiatry. 1986;149:98-103.

29. Whitehouse AM, Freeman CL, Annandale A. Body size estimation in anorexia nervosa. Br J Psychiatry. 1988;1553(2):23-6.

30. Thomas CD, Freeman RJ. Body-image marking. Validity of body-width estimates as operational measures of body image. Behav Modif. 1991;15(2):261-70.

31. Stewart A, Benson P, Michanikou E, Tsiota D, Narli M. Body image perception, satisfaction and somatotype in male and female athletes and non-athletes: results using a novel morphing technique. J Sports Sci. 2003;21:815-23.

32. Priebe $S$, Rohricht F. Specific body image pathology in acute schizophrenia. Psych Res. 2001;10:289-301.

33. Slade PD, Russel GSM. Awareness of body dimensions in anorexia nervosa: crosss-sectional and longitudinal study. Psychol Med. 1973;3:188-99.

34. Molinari E. Body size estimation in anorexia nervosa. Percept Mot Skills. 1995;81:23-33.

35. Thurm BE. Efeitos da dor crônica em atletas de alto rendimento em relação ao esquema corporal, agilidade psicomotora e estados de humor [dissertação]. São Paulo (SP): Universidade São Judas Tadeu; 2007. 